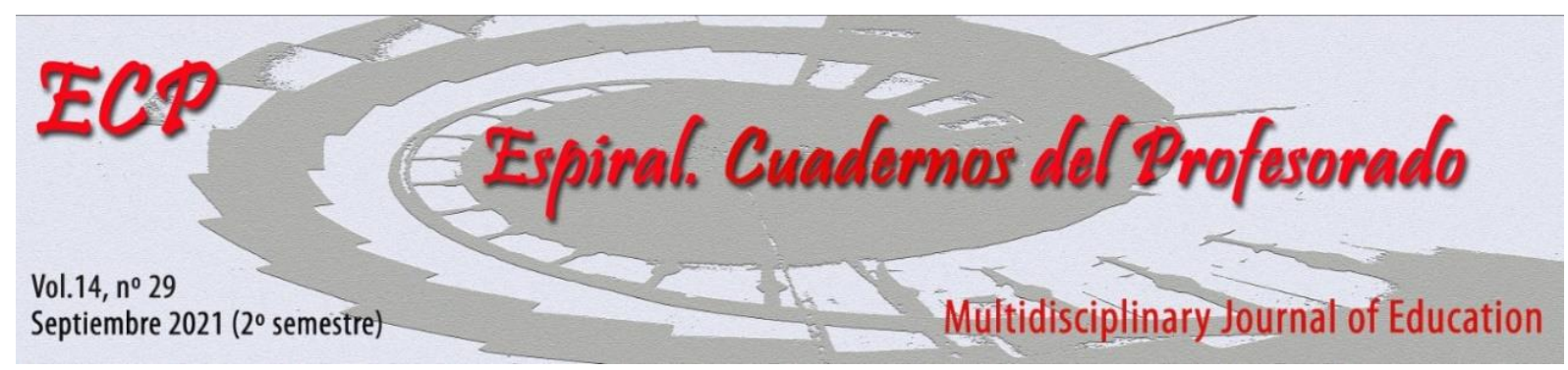

\title{
Experiencia didáctica en Educación Física para la mejora de actitudes hacia el cuidado del medio ambiente utilizando como recurso papel y cartón reciclado en futuros profesionales de la actividad física y deporte
}

\section{Didactic experience in Physical Education to improve attitudes towards caring for the environment using recycled paper and cardboard as a resource in future professionals of physical activity and sport}

\author{
Javier Arturo Hall-López \\ Facultad de Deportes, Universidad Autónoma de Baja California, México
}

\begin{abstract}
Resumen
Antecedentes: El cuidado al medio ambiente y la atención al cuerpo humano y la salud, son parte del perfil de egreso de la educación básica en México, esta experiencia, vincula ambos perfiles desde la educación física. Objetivo: Evaluar la actitud hacia el medio ambiente de futuros licenciados en actividad física y deporte que participaron en una experiencia didáctica capacitándose y aplicando un programa de educación física con intensidad moderada a vigorosa, utilizando como recurso didáctico papel y cartón reciclado en estudiantes de primaria. Metodología: 27 futuros licenciados en actividad física y deporte, participaron en el estudio, antes y después de la intervención se utilizó el cuestionario de actitudes hacia el medioambiente en la práctica de actividades físico-deportivas en el medio natural. Resultados: El análisis estadístico con test t-Student para muestras relacionadas, reportó diferencias significativas $(\mathrm{p}<0.05)$ en los valores promedio de actitud ambiental antes $3.5 \pm 0.6$ que después de la intervención $4.1 \pm 0.6$ con un porcentaje de cambio de $17.1 \Delta \%$. Conclusiones: En los estudiantes participantes la intervención fue efectiva para mejorar la actitud ambiental en educación física, favoreciendo los objetivos del desarrollo sostenible salud y bienestar, la educación de calidad, la producción y consumo responsables establecidos por la Organización de las Naciones Unidas (ONU).
\end{abstract}

Palabras clave: Actitud; Actividad Física; Educación Ambiental; Educación Física.

\begin{abstract}
Background: Care for the environment and care for the human body and health are part of the profile of graduation from basic education in Mexico, this experience links both profiles from physical education. Objective: To evaluate the attitude towards the environment of future graduates in physical activity and sport who participated in a didactic experience training and applying a physical education program with moderate to vigorous intensity, using recycled paper and cardboard as a teaching resource in primary school students. Methodology: 27 future graduates in physical activity and sports participated in the study. Before and after the intervention, the questionnaire on attitudes towards the environment was used in the practice of physical-sports activities in the natural environment. Results: The statistical analysis with Student's t-test for related samples, reported significant differences $(\mathrm{p}<0.05)$ in the average values of environmental attitude before $3.5 \pm 0.6$ than after the intervention $4.1 \pm 0.6$ with a change percentage of $17.1 \Delta \%$. Conclusions: In participating students the intervention was effective to improve the environmental attitude in physical education, favoring the objectives of sustainable development, health and well-being, quality education, responsible production and consumption established by the United Nations (UN).
\end{abstract}

Keywords: Attitude; Physical Activity; Environmental Education; Physical Education.

Fecha de recepción: 27/12/2020

Fecha de aceptación: 30/06/2021

Correspondencia: Javier Arturo Hall-López, Universidad Autónoma de Baja California, México Email: javierhall@uabc.edu.mx 


\section{Introducción}

Los objetivos de desarrollo sostenible (ODS) fueron establecidos por la Organización de las Naciones Unidas (ONU); para luchar contra la pobreza extrema en sus diversas dimensiones; dichos objetivos lógicamente, representan una llamado de atención para las distintas entidades que conforman la sociedad(Pérez Betancourt \& Betancourt Rodríguez, 2019),, como lo son las instituciones de educación superior (Hernández-Arteaga, Alvarado-Pérez y Luna, 2015; Ordóñez-Díaz, Montes-Arias y GarzónCortés, 2018), donde se han reestructurado los currículos de sus programas educativos y se han adecuado a las nuevas exigencias en el campo de la educación ambiental (Bravo Mercado, 2012). Para ello, se ha recomendado crear políticas y acciones de desarrollo sostenible que involucren a los estudiantes, los académicos y los administrativos (Armijo de Vega, Ojeda-Benítez, Ramírez-Barreto y QuintanillaMontoya, 2006), podrá contribuir al equilibrio ambiental mediante estrategias como el reciclaje de papel y cartón en la comunidad universitaria (Pellegrini Blanco y Reyes Gil, 2009).

En concordancia con este abordaje, la Universidad Autónoma de Baja California (UABC), en su Plan de Desarrollo Institucional (PDI 2019-2023), establece como parte de las políticas, estrategias y acciones institucionales a el cuidado del medio ambiente como el número 11, cuyo objetivo: promover entre la comunidad la cultura de respeto y cuidado del medio ambiente desde la perspectiva del desarrollo sostenible. Esta responsabilidad recae en la Coordinación General de Proyectos de Gestión Ambiental, la cual gestiona los esfuerzos en las diferentes unidades académicas de la UABC, entre las que se pueden mencionar la Facultad de Deportes UABC. Algunas de las innumerables misiones de esta facultad son la formación de profesionales competentes en la actividad física y en el deporte para coadyuvar al desarrollo de la sociedad a través de la cultura física.

En concordancia con este abordaje, en México la educación básica, como parte de sus ámbitos del perfil egreso establece a la atención al cuerpo y la salud y cuidado al medio ambiente, los cuales pueden ser abordados desde la educación física (SEP, 2017). Resultados de estudios educativos han determinado que el trabajo multidisciplinario y cooperativo, es fundamental para aplicar de manera exitosa programas de educación ambiental (Perales \& Gutiérrez Pérez, 2010), en los cuales pueden participar los profesores de educación física (Domingues, Kunz, \& Araújo, 2011), mediante diversas actividades que estimulen el aprovechamiento de materiales reciclados (Hall, et al., 2018a).

\section{Origen de la Experiencia}

En México, los problemas de sedentarismo se ven reflejados en la Encuesta Nacional de Salud y Nutrición de Medio Camino ENSANUT MC 2016, que reporta que el 17.2\% de la población de entre 1014 años de edad se categorizan como activos de acuerdo a los criterios de la Organización Mundial de la Salud (OMS) que en el plan de acción mundial sobre actividad física recomienda que niños, niñas y adolescentes dediquen como mínimo 60 minutos diarios en actividad física de intensidad moderada a vigorosa (OMS, 2019). Desde la perspectiva de la educación física se puede coadyuvar en la solución fomentando la actividad física de moderada a vigorosa por lo menos la mitad del tiempo que se decida a la clase de educación física (Hall, et al., 2017 (a)), acuerdo con estándares establecidos internacionalmente por el National Association for Sport and Physical Education (NASPE) (Baghurst, Langley, \& Bishop, 2015). Ya que en el contexto educativo mexicano investigaciones han analizado que la docencia impartida por el profesorado de educación física, promueve la participación del estudiantado en un 34 a $41 \%$ en actividad física moderada a vigorosa (Flores Moreno et al., 2016; Hall-López et al., 2017(b); Hall López et al., 2018 (b)).

La actitud del profesorado de educación física esta reportada como elemento de éxito en el proceso enseñanza aprendizaje (Fernández Rivas \& Espada Mateos, 2016), es entendida como un conjunto de percepciones, creencias, sentimientos a favor o en contra ante la postura educativa (Azcárraga, Pomes, \& Henríquez, 2013), y se puede aprovechar para combinar acciones educativas relativas a los objetivos del desarrollo sostenible establecidos por la Organización de las Naciones Unidas (ONU) abordando contenidos de salud y bienestar, la educación de calidad, la producción y consumo responsables (Pérez Betancourt \& Betancourt Rodríguez, 2019). 


\section{Objetivo}

Por lo anterior, la presente investigación abordo los dos perfiles de egreso: 1) cuidado del medio ambiente y 2) atención al cuerpo y la salud, planteados en el programa de educación básica vigente establecido por la Secretaría de Educación Pública (SEP, 2017), para implementar contenidos en educación física con intensidad moderada a vigorosa, utilizando como recurso didáctico papel y cartón reciclado, evaluando la actitud hacia el medio ambiente como experiencia didáctica en futuros licenciados en actividad física y deporte al realizar prácticas de educación física en educación primaria.

\section{Metodología}

La investigación se aprobó y registró en la Coordinación General de Posgrado e Investigación de la Universidad Autónoma de Baja California, bajo el protocolo número 149/1823.

En la experiencia didáctica participaron 27 estudiantes de quinto semestre de la licenciatura en actividad física y deporte con una edad entre los 19 y 26 años (15 hombres y 12 mujeres) de la Facultad de Deportes de la Universidad Autónoma de Baja California, que cursaban la unidad de aprendizaje obligatoria evaluación de la actividad física y deporte y realizaron prácticas de educación física en 27 grupos de quinto y sexto grado de educación primaria con estudiantes de una edad promedio de $10.5 \pm 4.3$ años (470 hombres y 482 mujeres), contando con la anuencia de participación a directivos y profesores de centros educativos públicos de la ciudad de Mexicali, Baja California, México, siguiendo los principios y ética de investigación, por lo anterior la participación fue voluntaria mediante carta de consentimiento por parte de los futuros licenciados en actividad física y deporte y el estudiantado del centro escolar participante (Rupali, 2005).

Las fases para la concreción de la experiencia didáctica, se abordaron bajo un diseño metodológico cuasi-experimental con muestreo no probabilístico por conveniencia (Thomas, Nelson, \& Silverman, 2015), donde la variable independiente, el programa de educación física con intensidad moderada a vigorosa utilizando como recurso didáctico papel y cartón reciclado fue manipulada durante su formación como licenciados en actividad física y deporte en la unidad de aprendizaje evaluación de la actividad física y deporte para medir su efecto sobre la variable dependiente actitud hacia el medio ambiente en cuatro fases.

El programa de educación física con intensidad moderada a vigorosa, utilizando como recurso didáctico papel y cartón reciclado, fue aplicado en la siguiente manera:

1.-La primera fase fue capacitar a los futuros licenciados en actividad física y deporte, en con contenidos Como generales sobre la contaminación en la ciudad de Mexicali Baja California, el marco jurídico estatal y federal respecto al manejo integral de residuos, ¿Qué es un residuo?, ¿Quiénes son generadores? y la clasificación optima de los residuos de papel y cartón. Evaluando previamente las actitudes hacia el medioambiente en la práctica de actividades físico-deportivas en el medio natural (Santos-Pastor, Cañadas, \& Martínez-Muñoz, 2019; Hall-López, \& Alarcón Meza, 2019).

2.-La segunda fase capacitar a los futuros licenciados en actividad física y deporte sobre la planeación y diseñar las secuencias didácticas para conducir las sesiones de educación física, utilizando como material papel y cartón reciclado, con estrategias y tareas que impliquen acciones motrices con el objetivo de promover y sensibilizar al alumno sobre la importancia en la en la generación de residuos (papel y cartón) y el impacto que este tiene en el medio ambiente además de concientizarlos en los procesos de reciclaje, reducción, clasificación y separación de residuos, identificar los señalamiento y su correcto depósito en las áreas de los centros escolares.

3.-La tercera fase fue practicar la experiencia didáctica de manera interna por parte de los futuros licenciados en actividad física y deporte, aplicando prácticas internas del programa tomando en cuenta el plan de estudios vigente de educación básica en el área de desarrollo personal y social y se adaptara a la educación física, en el componente pedagógico didáctico de desarrollo de la motricidad para trabajar el cuidado al medio ambiente utilizando como material didáctico papel reciclado. Los dos aprendizajes esperados fueron 1.-Combinar distintas habilidades motrices en retos individuales y cooperativos, para tomar decisiones y mejorar su actuación, 2.-Reconocer sus habilidades motrices en juegos que practican o 
Experiencia didáctica en Educación Física para la mejora de actitudes hacia el cuidado del medio ambiente utilizando como recurso papel y cartón reciclado

practicaban en su comunidad, estado o región para participar en distintas manifestaciones de la motricidad se realizaron 4 prácticas de 50 minutos de acuerdo al contexto educativo mexicano el plan de estudios vigente de educación básica (SEP, 2017). A partir de lo anterior, se organizaron contenidos por secuencias de tareas con estrategias que buscaron sensibilizar al alumno sobre la importancia en la generación de residuos utilizando como material didáctico papel y cartón reciclado, con estrategias didácticas en las que el profesor de manera intencionada fomentara movimientos en la clase de educación física para que el estudiantado realizara actividad física con intensidad moderada a vigorosa por lo menos la mitad del tiempo de la clase, acuerdo con estándares establecidos internacionalmente por el National Association for Sport and Physical Education (NASPE) (Baghurst, Langley, \& Bishop, 2015).

4.-La cuarta fase, fue aplicar la experiencia didáctica en ámbito educativo real, realizando 4 practicas por los futuros licenciados en actividad física y deporte, en clase de educación física en estudiantes de quinto y sexto de primaria con los contenidos por secuencias de tareas con estrategias que buscaron sensibilizar al alumno sobre la importancia en la generación de residuos utilizando como material didáctico papel y cartón reciclado, evaluando en el estudiantado antes y después de la intervención la actitud hacia el medio ambiente en la infancia en escolares (Francielli, et al., 2013). Y finalmente Evaluando en los futuros licenciados en actividad física y deporte las actitudes hacia el medioambiente en la práctica de actividades físico-deportivas en el medio natural (Santos-Pastor, Cañadas, \& MartínezMuñoz, 2019).

Para valorar la relación causa efecto, o el grado de cambio producido por el proceso educativo, estableciendo una relación causa efecto se evaluó antes y después de la intervención, dos cuestionarios referentes a la actitud ambiental en los futuros licenciados en actividad física y deporte y en los estudiantes de educación física al realizar sus prácticas docentes.

Escala de actitudes hacia el medioambiente en la práctica de actividades físico-deportivas en el medio natural, validado en estudiantes universitarios de titulaciones relacionadas con la actividad física y el deporte, el cuestionario cuenta con 16 ítems a responder en escala tipo Likert con 5 opciones de respuesta (1, Nada de acuerdo; 5 , Muy de acuerdo), la validación reportada del cuestionario obtuvo un alfa de Cronbach de 0.75. La escala aborda tres factores, que se definen como: (1) conocimiento sobre la educación ambiental y el impacto de la actividad física en el medio natural (ítems 1,6,7,9,12,14 y 16); (2) educación en temas sobre educación ambiental (ítems 2,3,4,5 y 8); y (3) actitudes propias durante la práctica de actividad física deportiva en el medio natural (ítems 10,11,13, y 15) (Santos-Pastor, Cañadas, \& Martínez-Muñoz, 2019).

Cuestionario de actitudes hacia el medio ambiente en la infancia en escolares (Francielli, et al., 2013), que abordan la dimensión compromiso real con el ambiente, y consta de 6 ítems a responder en escala tipo Likert de 0 al 4 ( $0=$ Nunca, 1=Casi Nunca, $2=$ A veces, $3=$ Casi Siempre4= Siempre). La validación reportada del cuestionario obtuvo variaciones entre .89 y .91 en alfa de Cronbach.

Para asegurar que en las sesiones de educación física se realizó actividad física moderada a vigorosa, se utilizó el Sistema para Observar el Tiempo de Instrucción de Actividad Física (SOFIT: System for Observing fitness and Instruction Time) (Mckenzie, Mckenzie, Sallis, \& Nader, 1992; Mckenzie, 2002); El instrumento evalúa al profesorado de manera cuantitativa, por medio de la actividad promovida en clase de educación física al estudiantado. La totalidad de las clases de educación física fueron evaluadas acorde a la metodología SOFIT, elegido aleatoriamente dos estudiantes hombres y dos estudiantes mujeres del grupo y observándolos en secuencia rotatoria de doce intervalos durante veinte segundos, repitiendo las observaciones durante toda la clase, siguiendo un audio que forma parte del instrumento de evaluación que indica, los tiempos de registro de la actividad. Para determinar la intensidad de las tareas motrices en las clases de educación física, se usaron códigos para clasificar la intensidad, los cuales permitieron estimar el gasto energético asociado con la actividad física, este procedimiento acorde al protocolo SOFIT se clasificó en cinco códigos: 1.- acostado, 2.- sentado, 3.- parado, 4.- caminando, y 5.- muy activo que corresponde a correr o una actividad con un gasto energético mayor. A partir de la cuantificación de estos códigos se estableció la actividad física moderada a vigorosa sumando porcentualmente los códigos 4.caminando y 5.- muy activo del total del tiempo de las clases de educación física. La confiabilidad inter evaluadores, con conocimiento en los procedimientos técnicos del instrumento y memorizaron las 
definiciones operativas de los códigos referentes al contexto y actividad física, resulto con un valor Kappa .86. (Szklo, \& Nieto, 2007).

\section{Repercusión sobre la Comunidad Educativa}

El análisis estadístico se realizó con el software estadístico para las ciencias sociales (SPSS), versión 25, calculando los valores descriptivos de la intensidad en la clase de educación física y las variables del cuestionario de actitud ambiental; y el porcentaje de diferencia $(\Delta \%)$ ([(Media-2 - Media1)/Media-1] x 100) (Vincent, 2012). Para verificar la normalidad de los grupos y homogeneidad de la varianza de los datos, se utilizó el test estadístico de Kolmogorov-Smirnov, presentando un grado de significancia de $\mathrm{P}$-Valor < 0.05; Como estudio longitudinal, la estadística inferencial comparo las variables de actitud ambiental antes y después de la experiencia didáctica utilizando la prueba t Student para muestras relacionadas como estadística inferencial, con la finalidad de calcular la igualdad de la varianza, determinando un nivel de $\alpha \leq 0.05$, es decir un $5 \%$ como porcentaje de error de la prueba estadística.

\section{Figura 1}

Valores de actitudes hacia el medioambiente en la práctica de actividades físico-deportivas en el medio natural en los futuros licenciados en actividad física y deporte, antes y después de estudiantes de participar en el programa de Educación Física con intensidad moderada a vigorosa, utilizando como recurso didáctico papel y cartón reciclado.

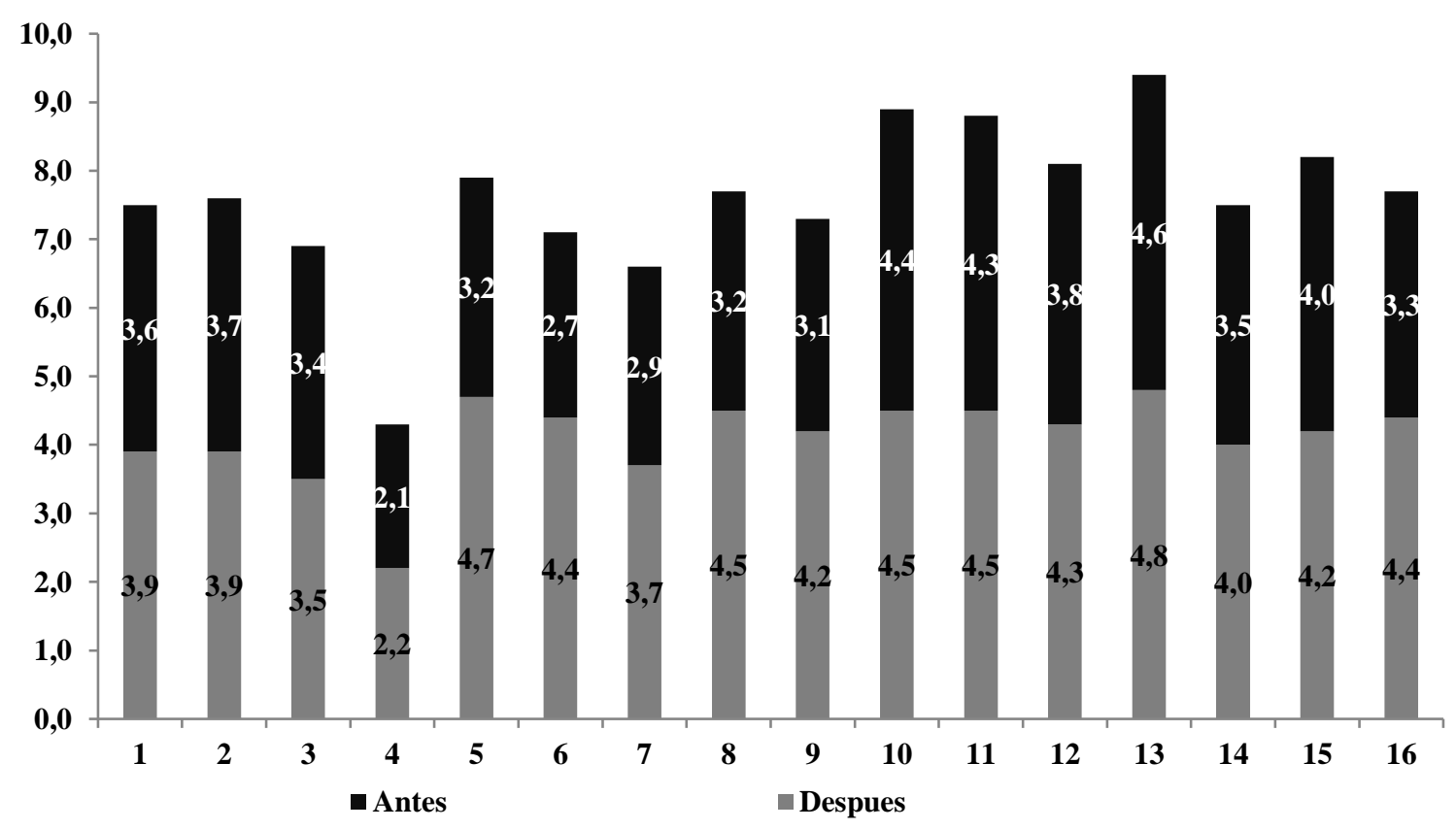

En la Tabla 1, se observa el cálculo de en los porcentajes de cambio $(\Delta \%)$ de actitudes de los futuros licenciados en actividad física y deporte antes y después de participar en el programa De Educación Física con intensidad moderada a vigorosa. 
Experiencia didáctica en Educación Física para la mejora de actitudes hacia el cuidado del medio ambiente utilizando como recurso papel y cartón reciclado

\section{Figura 2}

Valores de actitud ambiental del estudiantado (ítem 1 al 6) antes y después de estudiantes de quinto y sexto de primaria al participar en el programa de Educación Física con intensidad moderada a vigorosa, utilizando como recurso didáctico papel y cartón reciclado.

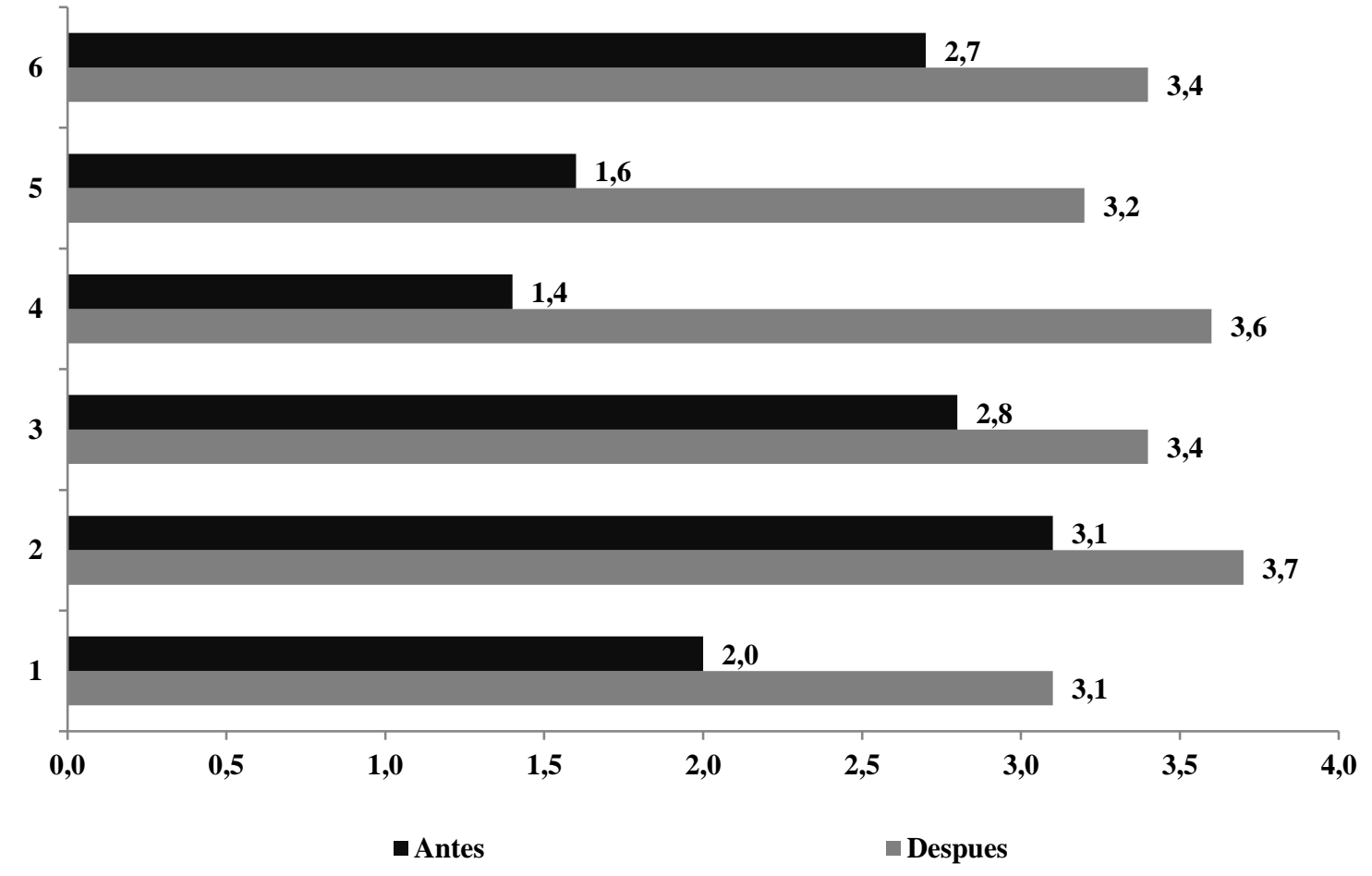

\section{Tabla 1}

Cálculo de en los porcentajes de cambio ( $4 \%$ ) de actitudes antes y después de la intervención en futuros licenciados en actividad física y deporte.

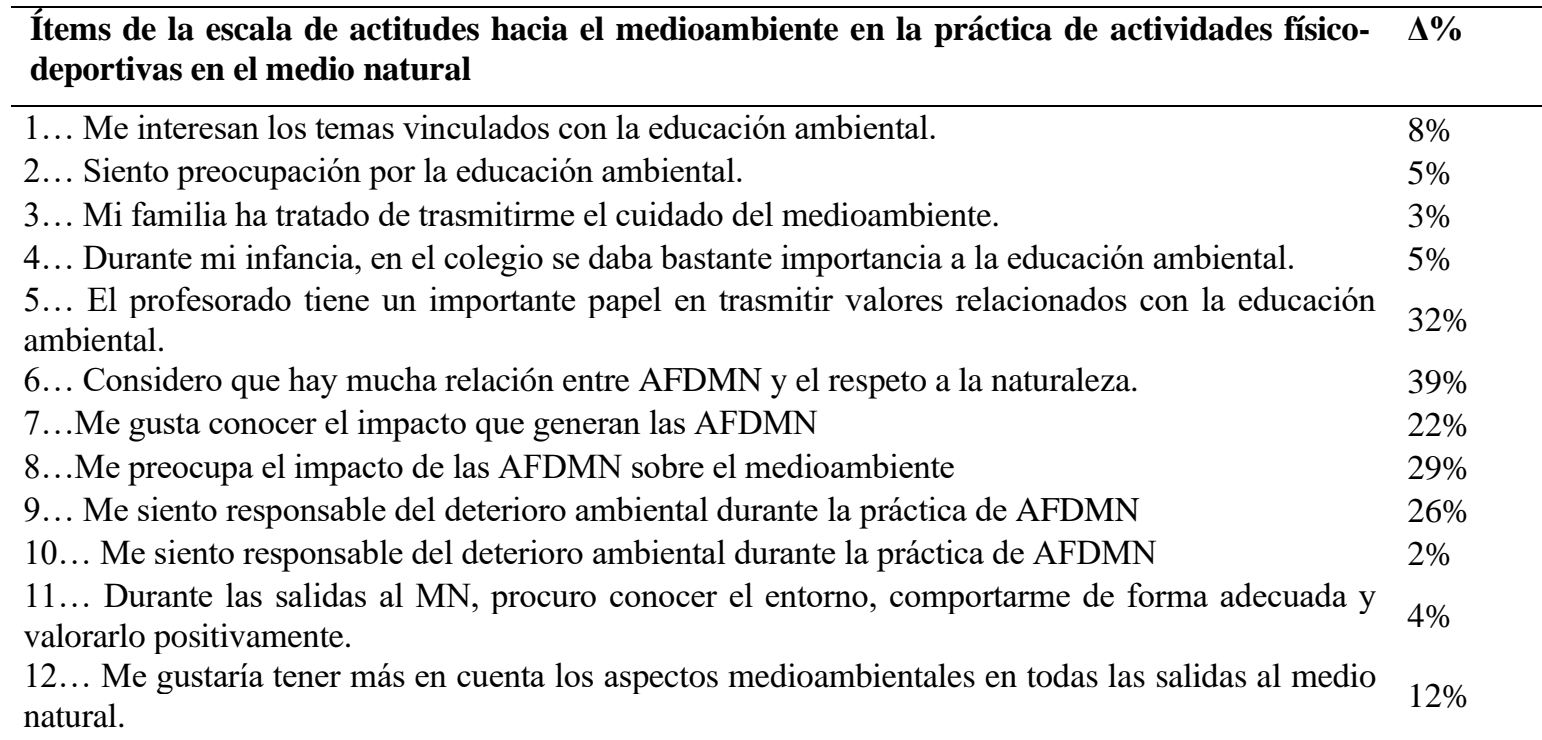


13... Durante las salidas al MN no me gusta que se deje basura en el entorno.

$4 \%$

$13 \%$

14... Me gusta analizar la repercusión que tienen mis AFDMN sobre el medioambiente.

$5 \%$ la naturaleza.

16... Me gustaría implicarme con un grupo que trabaje las AFDMN y el medioambiente.
$25 \%$

En la Tabla 2, se observa el cálculo de en los porcentajes de cambio $(\Delta \%)$ de actitudes del estudiantado antes y después de participar en el programa de educación física con intensidad moderada a vigorosa.

Tabla 2

Cálculo de en los porcentajes de cambio (4\%) de actitudes en los estudiantes de quinto y sexto grado antes y después de la intervención.

\begin{tabular}{ll}
\hline Ítems del cuestionario actitudes hacia el medio ambiente en la infancia en escolares & $\mathbf{\Delta \%}$ \\
\hline 1... Hablas con tus padres acerca de cómo ayudar a resolver los problemas ambientales. & $55 \%$ \\
2... Cierra la llave mientras te cepillas los dientes para ahorrar agua. & $19 \%$ \\
3... Para ahorrar energía, apagas las luces de la casa cuando no se la utiliza. & $21 \%$ \\
4... Le pides a tu familia que recicle algunas de las cosas que no utilizan. & $157 \%$ \\
5... Preguntas a otras personas qué se puede hacer para ayudar a reducir la contaminación. & $100 \%$ \\
6... Cierra la puerta del refrigerador mientras decide qué agarrar. & $26 \%$ \\
\hline
\end{tabular}

\section{Evaluación}

El principal resultado de la concluir la experiencia didáctica fue las actitudes hacia el medioambiente en la práctica de actividades físico-deportivas en el medio natural en los futuros licenciados en actividad física y deporte, mejoraron en un $17.1 \Delta \%$, con valores promedio de $3.5 \pm 0.6$ antes respecto a $4.1 \pm 0.6$ después de la intervención, el análisis estadístico con test t-Student, para muestras relacionadas reportó diferencias significativas $(\mathrm{p}<0.05)$.

En promedio los ítems del factor 1, relativo al conocimiento sobre la educación ambiental y el impacto de la actividad física en el medio natural (ítems 1, 6, 7, 9, 12, 14 y 16); el porcentaje de cambio fue $20 \Delta \%$ antes 3.3 y 4.1 después de la intervención. El factor 2 , relativo a la educación en temas sobre educación ambiental (ítems 2, 3, 4, 5 y 8); resulto con un porcentaje de cambio fue $15 \Delta \%$ antes 3.3 y 3.9 después de la intervención. El factor 3, relativo a las actitudes propias durante la práctica de actividad física deportiva en el medio natural (ítems $10,11,13$, y 15) el porcentaje de cambio resulto $4 \Delta \%$ antes 4.3 y 4.5 después de la intervención (Santos-Pastor, Cañadas, \& Martínez-Muñoz, 2019).

$\mathrm{Al}$ evaluar con el cuestionario de actitudes hacia el medio ambiente en la infancia en escolares (Francielli, et al., 2013), al estudiantado de educación física de quinto y sexto de primaria que participaron en las sesiones con intensidad moderada a vigorosa, utilizando como recurso didáctico papel y cartón reciclado dirigidas por los futuros licenciados en actividad física y deporte el resultado fue que mejoró la actitud hacia el medio ambiente en un $47.8 \Delta \%$, el análisis estadístico con test t-Student, para muestras relacionadas reportó diferencias significativas $(\mathrm{p}<0.01)$ en los valores promedio de actitud antes $2.3 \pm 0.7$ que después de la intervención $3.4 \pm 0.2$.

La investigación, atendió las directrices del programa de educación básico en el contexto mexicano (SEP, 2017) y como innovación educativa atendió las recomendaciones de la Organización Mundial de la Salud (OMS) en cuanto a la práctica de actividad física de intensidad moderada a vigorosa (OMS, 2019). La estadística descriptiva de la intensidad en las clases de educación física evaluadas mediante SOFIT, resulto con un con una actividad física moderada a vigorosa, promedio de 54 $\pm 3.8 \%$, lo cual se encuentra dentro de los estándares establecidos internacionalmente por el National Association for Sport and Physical Education (NASPE) (Baghurst, Langley, \& Bishop, 2015).

Debido a las características multidisciplinarios de la educación ambiental su medición y evaluación se ha considerado compleja (Páramo, 2017), de igual manera se ha reportado que el éxito de la conducta ambiental esta cohesionado en conocimientos, actitudes y valores (Torres Rivera, et al., 2018), 
y se establece que las actitudes influyen de manera significativa en el entorno escolar entre los amigos, para en su conducta ambiental (Cortes et al., 2017); en ese sentido en el campo educativo de la educación física se ha identificado al profesorado con un adecuado rol para generar hábitos de práctica de actividad física y en general los escolares, que manifiestan en su figura una imagen positiva (Cárcamo Oyarzún, 2018), en ese sentido, es importante la adecuación curricular desde la formación docente en educación física para el trabajo multidisciplinario hacia la educación ambiental (Domingues, Kunz, \& Araújo, 2011), para la conducción de contenidos, estrategias didácticas y diseño de tareas motrices en educación física, que atienda una alfabetización corporal combinada con en los objetivos de la Organización de las Naciones Unidas para la Educación UNESCO (Bolanos-Motta, et al. 2018; Frizzo, \& Souza, 2019).

Desde la formación del profesorado investigaciones consideran que desde la formación universitaria se puede fomentar la actividad física moderada a vigorosa en los futuros licenciados en actividad física y deporte (Hall López et al., 2016; Hall-López, Sáenz-López Buñuel, \& Almagro, 2020; Hall-López, 2021), tal como se realizó en el presente estudio, al enfocar la educación física con la educación ambiental (Hall-López, et al., 2018 (a)), adecuándose a los diferentes contextos en centros educativos rurales y urbanos, en los cuales se ha demostrado diferencias en la manera de aprovechar los recursos para la educación física (Salazar et al., 2016; Hall-López, 2020) así como también en entornos virtuales y a distancia que favorezcan la educación ambiental mediante el movimiento (Hall-López, \& Ochoa-Martínez, 2020; Hall-López, Ochoa-Martínez, \& Alarcón Meza, 2021).

En la experiencia didáctica identificamos limitaciones, el diseño del estudio se adecuo a un contexto educativo geográfico especifico respetando el programa educativo, la investigación no conoto con grupo control para contrastar los resultados, aun sin embargo los instrumentos utilizados, para evaluar la intensidad de la actividad física y actitud ambiental fueron confiables, y consideramos que son de sencilla reproducibilidad para intervenciones de similar abordaje metodológico. La información derivada de este estudio contribuye al conocimiento del profesorado de educación física, para el futuro desarrollo de intervenciones efectivas en torno a la educación ambiental combinando la práctica de actividad física recomendada para la salud en clase de educación física.

Financiación: "Esta investigación fue financiada por la Universidad Autónoma de Baja California, bajo el protocolo número 149/1823”.

Conflicto de Intereses: "El autor declara que no tienen conflicto de intereses".

\section{Referencias}

Armijo de Vega, C., Ojeda-Benítez, S., Ramírez-Barreto, E. y Quintanilla-Montoya, A. (2006). Potencial de reciclaje de los residuos de una institución de educación superior: el caso de la Universidad Autónoma de Baja California. Ingeniería, 10(3), 13-21. https://www.redalyc.org/articulo.oa?id=467/46710302

Azcárraga, M., Pomes, M., \& Henríquez, S. (2013). Actitud de los profesores hacia la inclusión educativa. Papeles de trabajo-Centro de Estudios Interdisciplinarios en Etnolingüística y Antropología Socio-Cultural, 25: 51-59. https://core.ac.uk/download/pdf/61700917.pdf

Baghurst, T., Langley, J., \& Bishop, J. (2015). Physical Educators' Perceptions of Their Use of NASPE Standards. The Physical Educator, 72(5), 324-341. https://doi.org/10.18666/TPE-2015-V72-I5-6194

Bolaños Motta, J., Pérez Rodríguez, M., \& Casallas Forero, E. (2019). Alfabetización Corporal. Una propuesta de aula desde la psicomotricidad. Estudios Pedagógicos, 44(3), 23-34. https://doi:10.4067/S071807052018000300023

Bravo Mercado, M. T. (2012). La UNAM y sus procesos de ambientalización curricular. Revista Mexicana de Investigación Educativa, 17(55), 1119-1146. https://www.redalyc.org/articulo.oa?id=140/14024273006

Cárcamo Oyarzún, J. (2018). El profesor de Educación Física desde la perspectiva de los escolares. Estudios Pedagógicos, 38(1), 105-119. 10.4067/S0718-07052012000100006

Cortes, F., Cabana Villca, R., Vega Toro, D., Aguirre Sarmiento, H., \& Muñoz Gómez, R. (2017). Variables influyentes en la conducta ambiental en alumnos de unidades educativas, región de CoquimboChile. Estudios Pedagógicos, 43(2), 26-46. 10.4067/S0718-07052017000200002 
Domingues, S. C., Kunz, E. \& Araújo, L. C. (2011). Educação ambiental e educação física: possibilidades para a formação de professores. Revista Brasileira de Ciências do Esporte, 33(3), 559571. https://dx.doi.org/10.1590/S0101-32892011000300003

Flores Moreno, P. J., Salazar, C. M., Gómez Figueroa, J. A., Barreto Villa, Y., Valdovinos González, O., Vicente Rivera, J. U., \& Del Río Valdivia, J. E. (2016). Medición del tiempo efectivo de la clase de educación física y su impacto en el gasto calórico en escolares de nivel primaria del municipio de Colima, México. Sportis. Scientific Journal of School Sport, Physical Education and Psychomotricity, 3(1), 3449. https://doi.org/10.17979/sportis.2017.3.1.1766

Francielli, G., Bolzan de Campos, C., Bedin, L. M., \& Castellá Sarriera, J. (2013). Actitudes hacia el medio ambiente en la infancia: un análisis de niños del sur de Brasil. Revista Latinoamericana de Psicología, 45(3), 459-471. https://dx.doi.org/10.14349/rlp.v45i3.1487

Fernández Rivas, M., \& Espada Mateos, M. (2016). Actitud del profesorado de educación física frente al $\begin{array}{llll}\text { aprendizaje } & \text { Movimento, } & \text { 80operativo. }\end{array}$ https://www.redalyc.org/articulo.oa?id=1153/115347695014

Frizzo, G., \& Souza, M. D. S. (2019). Educação física nas diretrizes da unesco: o paradigma da aptidão física e da saúde na formação do capital humano. Movimento (ESEFID/UFRGS), Porto Alegre, 25(10), 1-12. doi: https://doi.org/10.22456/1982-8918.76037

Hall-López, J. (2021). Programa de formación educativa para aumentar el índice de actividad física moderada a $\begin{array}{lllll}\text { vigorosa en profesores de } & \text { 192-199. }\end{array}$ https://doi.org/10.47197/retos.v0i39.78014

Hall-López, J. A., Ochoa-Martínez, P. Y., \& Alarcón Meza, E. I. (2021). Alternativas durante el covid-19, para profesorado universitario dedicado a la enseñanza de la educación física. Cuerpo, Cultura Y Movimiento, 11(1), 4-13. https://doi.org/10.15332/2422474X.6464

Hall-López, J., \& Ochoa-Martínez, P. (2020). Enseñanza virtual en educación física en primaria en México y la pandemia por COVID-19. Ciencias De La Actividad Física UCM, 21(2), 1-7. https://doi.org/10.29035/rcaf.21.2.4

Hall-López, J. A. (2020). Rural-Urban differences in perception of effort and physical activity of adolescents in $\begin{array}{lllll}\text { physical education. } & \text { Revista } & \text { Espacios, } & \text { 41(26) }\end{array}$ http://ww.revistaespacios.com/a20v41n26/a20v41n26p29.

Hall-López, J. A., Sáenz-López Buñuel, P. \& Almagro, B. (2020). Actividad Física Moderada a Vigorosa en Educación Física. Kronos Revista Científica de Actividad Física y Deporte, 19(1), 1-2. https://revistakronos.info/articulo/actividad-fisica-moderada-a-vigorosa-en-educacion-fisica-2777-saq5ef3f1a1924bd

Hall-López, J. A., \& Alarcón Meza, E. I. (2019). Análisis de la intensidad en la adecuación curricular de educación física mediante reciclaje de papel: estudio de caso., En F. Santillán (coord.), El pensamiento del profesor investigador vínculo entre la teoría y la práctica, (pp. 181-190). Centro de Estudios e Investigaciones para el Desarrollo Docente CENID. http://www.cenid.org.mx/libros/libros19/libro014/\#p=1

Hall-López, J. A., Ochoa-Martínez, P. Y., Saavedra Maldonado, J. M., Villafranco Ruiz, C. G., Carboni Álvarez, C. y Cabrera González, J. L. (2018a). Promoción del cuidado del medio ambiente en educación física primaria utilizando papel reciclado. En Muñoz Fernández, T. V. (ed.), Actualidades en actividad física y Deporte (pp. 187-211). Universidad de Guadalajara.

Hall Lopez, J. A., Ochoa-Martínez, P. Y., Macías Castro, R. A., Zúñiga Burruel, R., \& Sáenz-López Buñuel, P. (2018b). Actividad física moderada a vigorosa en educación física y recreo en estudiantes de primaria y secundaria de la frontera México-USA. Sportis. Scientific Journal of School Sport, Physical Education and Psychomotricity, 4(3), 426-442. https://doi.org/10.17979/sportis.2018.4.3.3175

Hall-López, J. A., Ochoa-Martínez, P. Y., Zamudio Bernal, A., Sánchez León, R., Uriarte Garza, L., Almagro, B., Moncada-Jiménez, J. \& Sáenz-López Buñuel, P. (2017a). Efecto de un programa de actividad física de moderada a vigorosa de diez meses sobre el vo2máx y el porcentaje de grasa corporal en niños con sobrepeso y obesidad. MHSalud: Revista en Ciencias del Movimiento Humano y Salud, 14(1), 1-14. http://dx.doi.org/10.15359/mhs.14-1.6

Hall-López, J., Ochoa-Martínez, P., Zuñiga Burruel, R., Alarcón Meza, E., Macías Castro, R., \& Sáenz-López Buñuel, P. (2017b). Moderate-to-vigorous physical activity during recess and physical education among mexican elementary school students (Actividad física moderada a vigorosa durante el recreo y clase de 
Experiencia didáctica en Educación Física para la mejora de actitudes hacia el cuidado del medio ambiente utilizando como recurso papel y cartón reciclado

educación física en niños mexicanos de escuela primaria). Retos, 31, 137-139. https://doi.org/10.47197/retos.v0i31.49640

Hall López, J. A., Ochoa-Martínez, P. Y., González Terrazas, J. C., \& González Ramírez, J. R. (2016). Duración, intensidad y contexto de las clases de Educación Física impartidas por profesores de Educación Física y estudiantes de licenciatura. Sportis. Scientific Journal of School Sport, Physical Education and Psychomotricity, 3(1), 63-82. https://doi.org/10.17979/sportis.2017.3.1.1723

Hernández-Arteaga, R., Alvarado-Pérez, J. y Luna, J. (2015). Responsabilidad social en la relación universidadempresa-Estado. Educación y Educadores, 18(1), 95-110. https://dx.doi.org/10.5294/edu.2015.18.1.6

Licenciatura en Actividad Física y Deporte Universidad Autónoma de Baja California (UABC) (2018). Plan de estudio 2012-2. http://deportes.uabc.mx/index.php/programas-educativos/licenciatura-en-actividadfisica/plan-2012-2/u-aprendizaje

Mckenzie, T. (2002). SOFIT. System for Observing Fitness Instruction Time. Overview and Training Manual. San Diego State University. https://thomckenzie.com/useful-tools/observation/

Mckenzie, T., Sallis, J., \& Nader, P. (1992). Sofit-System for Observing Fitness Instruction Time. Journal of Teaching in Physical Education, 11(2), 195-205. https://doi.org/10.1123/jtpe.11.2.195

Medina, C., Jáuregui, A., Campos-Nonato, I., Barquera, S. (2018). Prevalencia y tendencias de actividad física en niños y adolescentes: resultados de Ensanut 2012 y Ensanut MC 2016. Salud Pública de México, 60(3), 263-271. http://saludpublica.mx/index.php/spm/article/view/8819.

Misión de la Facultad de Deportes de la Universidad Autónoma de Baja California (UABC) (2018). Plan de desarrollo de la unidad académica. http://deportes.uabc.mx/index.php/sobre-nosotros/mision-vision.

Modelo educativo de la Universidad Autónoma de Baja California (UABC) (2018). Principios orientadores: alumnos. Recuperado de http://www.uabc.mx/formacionbasica/modeloedu.html.

Ordóñez-Díaz, M., Montes-Arias, L., \& Garzón-Cortés, G. (2018). Importancia de la educación ambiental en la gestión del riesgo socio-natural en cinco países de América Latina y el Caribe. Revista Electrónica Educare, 22(1), 345-363. https://dx.doi.org/10.15359/ree.22-1.17

Organización Mundial de la Salud OMS (2019). Plan de acción mundial sobre actividad física 2018-2030: personas más activas para un mundo más sano. https://apps.who.int/iris/handle/10665/327897

Páramo, P. (2017). Reglas proambientales: una alternativa para disminuir la brecha entre el decir-hacer en la educación ambiental. Suma Psicológica, 24(1), 42-58. https://dx.doi.org/10.1016/j.sumpsi.2016.11.001

Pellegrini, B. N. y Reyes Gil, R. (2009). Reciclaje de papel en la Universidad Simón Bolívar. Revista de Investigación, (67), 45-57. http://revistas.upel.digital/index.php/revinvest/article/view/3812/1888

Perales, F., \& Gutiérrez Pérez, J. (2010). Claves de cooperación interdisciplinar: una visión retrospectiva de la educación ambiental desde su trayectoria en la Universidad de Granada. Revista Eureka sobre Enseñanza y Divulgación de las Ciencias, 7, 400-412.

Pérez Betancourt, A., \& Betancourt Rodríguez, M. Z. (2019). El enfoque de género desde la formación docente y su relación con la ciencia, la tecnología y la sociedad. Revista Boletín Redipe, 8(3), 200-208. https://revista.redipe.org/index.php/1/article/view/713

Plan de Desarrollo Institucional de la Universidad Autónoma de Baja California (UABC) 2019-2023. (2019). http://www.uabc.mx/planeacion/pdi/2019-2023/PDI_2019-2023.pdf.

Rupali, G. (2005). Research involving children: regulations, review boards and reform. Journal of Health Care Law and Policy, 8(2), 264-330. https://digitalcommons.law.umaryland.edu/jhclp/vol8/iss2/6

Salazar, C. M., Flores Moreno, P. J., Barajas Pineda, L. T., Medina Valencia, R. T., Chávez Valenzuela, M. E., \& Camacho Acevedo, E. (2017). Evaluación del tiempo efectivo de la clase de educación física en escolares del ámbito rural. Revista Edu-Física, 9(19). http://revistas.ut.edu.co/index.php/edufisica/article/view/1097

Santos-Pastor, M. L., Cañadas, L., \& Martínez-Muñoz, L. F. (2019). Scale for Environmental Attitude in Physical Activities in Natural Environments. Apunts. Educación Física y Deportes, 137, 43-55. doi:10.5672/apunts.2014-0983.es.(2019/3).137.04

Secretaría de Educación Pública SEP (2017). Aprendizajes clave para la educación integral, educación física, educación básica. Plan y programas de estudio y sugerencias de evaluación. Ciudad de México. https://www.planyprogramasdestudio.sep.gob.mx/index-descargas-LMP-basica-edu-fisica.html

Szklo, M., \& Nieto J. (2007). Epidemiology: beyond the basics (2nd ed.) Jones \& Bartlett Learning.

Espiral. Cuadernos del Profesorado | ISSN 1988-7701 | 2021, 14(29), 113-123 
Thomas, J., Nelson, J., \& Silverman, S. (2015). Research Methods in Physical Activity (7th. Ed.). Human Kinetics. Human Kinetics.

Torres Rivera, L., Benavides Peña, J., Latoja Vollouta, C., \& Novoa Contreras, E. (2018). Presencia de una Educación Ambiental basada en conocimiento, actitudes y prácticas en la enseñanza de las ciencias naturales en establecimientos municipales de la ciudad de Los Ángeles, Chile. Estudios Pedagógicos, 43(3), 311-323. doi:10.4067/S0718-07052017000300018

Vincent, W. (2012). Statistics in kinesiology (4nd Ed.). Human Kinetics. 